\title{
L'Amazonie depuis la pénombre. Au sujet de trois séries photographiques de Musuk Nolte
}

From the shadows. On three series of photographs of Amazonia by Musuk Nolte La Amazonía desde la penumbra. Sobre tres series fotográficas de Musuk Nolte

\section{Morgana Herrera}

\section{OpenEdition}

\section{Journals}

Édition électronique

URL : http://journals.openedition.org/ideas/4749

DOI : $10.4000 /$ ideas.4749

ISSN : $1950-570$

Éditeur

Institut des Amériques

\section{Référence électronique}

Morgana Herrera, «L'Amazonie depuis la pénombre. Au sujet de trois séries photographiques de

Musuk Nolte », IdeAs [En ligne], 13 | 2019, mis en ligne le 01 mars 2019, consulté le 19 avril 2019. URL http://journals.openedition.org/ideas/4749; DOI : 10.4000/ideas.4749

Ce document a été généré automatiquement le 19 avril 2019

\section{c) $(7)$}

IdeAs - Idées d'Amériques est mis à disposition selon les termes de la licence Creative Commons Attribution - Pas d'Utilisation Commerciale - Pas de Modification 4.0 International. 


\section{L'Amazonie depuis la pénombre. Au sujet de trois séries photographiques de Musuk Nolte}

From the shadows. On three series of photographs of Amazonia by Musuk Nolte La Amazonía desde la penumbra. Sobre tres series fotográficas de Musuk Nolte

Morgana Herrera

\section{Introduction}

Depuis une quinzaine d'années, on peut constater dans le circuit culturel péruvien une multiplication d'expositions réunissant des projets ayant trait à l'Amazonie ${ }^{1}$. Territoire d'intégration tardive à l'État-nation, la forêt amazonienne n'était guère présente dans l'imaginaire national péruvien qui s'est construit principalement sur la valorisation du passé inca, et donc sur la région andine ${ }^{2}$. Si certains critiques parlent péjorativement de mode, nul ne peut contester aujourd'hui une visibilité et un dynamisme amazonien sans précédent sur la scène artistique locale. Dans le cas spécifique de la photographie, on ne peut pas parler d'un phénomène nouveau tant l'Amazonie a été un terrain de prédilection des photographes depuis la fin du XIX ${ }^{e}$ siècle, que l'on songe à l'outil photographique chez les anthropologues, aux reportages du National Geographic ou aux publications du gouvernement péruvien pour la promotion du tourisme. Cependant, deux tendances plus récentes ont dominé le circuit d'expositions péruvien : d'une part, le public a assisté à une redécouverte de photographies historiques provenant d'albums de missions d'exploration, de missions religieuses et de cartes postales datant de la fin du XIX et du début du $\mathrm{xx}^{\mathrm{e}}$ siècle ${ }^{3}$; d'autre part, les photographes contemporains ont fait découvrir une Amazonie urbaine, adoptant des couleurs très vives et se revendiquant d'une esthétique tropicale, ou alors ont renouvelé les façons de représenter les peuples indigènes, dans des mises en scène émanant d'une esthétique réaliste magique ${ }^{4}$. Ces expositions n'ont pas seulement circulé à l'intérieur du Pérou, elles se sont également exportées à l'étranger, et ce malgré une action culturelle extérieure péruvienne qui demeure faible. Réunissant 
toutes ces tendances, l'exposition « Dans le pays des Amazones : 150 ans de photographie amazonienne » s'est tenue à Lima du 12 avril au 31 mai 2017, avant de continuer à circuler à travers le pays.

Dans ce panorama général, le travail du photographe Musuk Nolte est à distinguer : si sa photographie se différencie clairement de cette esthétique dominante, il fait systématiquement partie des expositions collectives sur l'Amazonie. À un moment de transition dans la photographie représentant la réalité amazonienne, où l'on s'éloigne des reportages documentaires «classiques» sur la faune, la flore ou les communautés indigènes, Nolte choisit les sujets les moins documentés malgré le boom des dernières années. Paradoxalement, les procédés artistiques qui rendent sa photographie très reconnaissable, comme le noir et blanc extrêmement contrasté et le flou, ne sont utilisés que dans sa photographie documentaire tandis que nous ne trouvons guère d'effets de style marqués dans ses projets artistiques ou conceptuels. Ainsi, s'il n'est certainement pas nouveau de mêler pratique documentaire et artistique, sa façon de les combiner et son choix particulier de sujets, permettent d'aller à l'encontre des images stéréotypées de la forêt tropicale dans une mise en récit particulière de cet espace et de ses habitants.

\section{Musuk Nolte dans le contexte du renouveau de la représentation de l'Amazonie péruvienne}

Le parcours de Musuk Nolte nous éclaire quant à sa pratique et sa situation privilégiée dans le milieu de la photographie contemporaine péruvienne. Né à Mexico en 1988, Nolte a fait ses études au Centre de l'Image de Lima, l'unique institut d'études supérieures du pays à proposer une formation entièrement consacrée à la photographie. La scène photographique péruvienne contemporaine est d'ailleurs encore loin d'être consolidée. La biennale de photographie de Lima n'a connu que deux éditions en 2012 et 2014 avant de disparaitre ; la galerie de photographie Secuencia, qui a posé un important jalon sur la scène locale, n'a existé que de 1977 à 1980 et, sur les deux uniques galeries qui aujourd'hui se consacrent exclusivement à la photographie, une seule possède un espace physique et dépend du Centre de l'Image. Ce tableau est cependant à nuancer : le Musée d'Art de Lima (MALI) a ouvert en 2006 sa première section photographique permanente ; la foire Lima Photo a tenu sa $8^{\text {e }}$ édition en 2017 et le photographe de mode internationalement reconnu, Mario Testino, a ouvert à Lima sa fondation MATE en 2012. Dans ce contexte mitigé, Musuk Nolte s'est surtout fait connaître grâce à son succès à l'étranger, garantie toujours efficace de se faire une place dans le panorama culturel péruvien. Il a commencé très jeune à travailler pour le quotidien national $\mathrm{El}$ Comercio avant de renoncer à ses activités comme photojournaliste pour se tourner vers la photographie d'entreprise et de contenus éditoriaux en fondant l'atelier Bellavista, activité qui est aujourd'hui sa principale source de revenu. Grâce à sa photographie artistique, il a longtemps été représenté par une des plus prestigieuses galeries d'art de Lima, la galerie Lucía de la Puente. La galeriste le définit d'ailleurs comme un photographe de l'hybride :

Son travail se situe entre la photographie documentaire et l'art. La qualité de ses images en noir et blanc avait déjà révélé son grand talent pour la capture et le dévoilement de la réalité ; mais ce sont ses travaux personnels, nombre d'entre eux abstraits, qui lui ont valu la reconnaissance comme un artiste total par le milieu ${ }^{5}$. 
En plus de huit expositions individuelles, de sa participation à de nombreuses biennales de photo, Musuk Nolte est devenu un incontournable des expositions collectives de photographes péruviens à l'étranger ${ }^{6}$. Il a, par ailleurs, reçu de nombreux prix, dont le premier prix du concours Juan Bartolomé de photographie humanitaire pour sa série Shawi en 2012 et a été récemment boursier de l'Emergency Fund de la fondation Magnum ainsi que de l'Elliott Erwit Havana Club 7 Fellowship. Ses photographies ont intégré les collections les plus prestigieuses du Pérou, comme celle du MALI. Nolte se consacre par ailleurs à l'édition de livres-photos et il a fondé en 2014 la maison d'édition KWY (qaway, « voir » en quechua). Comprendre les multiples facettes de Musuk Nolte permet de mieux entrer dans ses séries de documentaires photographiques sur l'Amazonie, en prenant en compte le dynamisme récent lié à cet espace et la construction qu'en fait un jeune photographe, ainsi que sa façon de se situer par rapport aux expositions collectives. De façon globale, la variété des séries amazoniennes de Nolte semble rompre un des grands écueils de toute représentation de ce territoire, celui d'une vision unique et simplificatrice d'une jungle sans nuances.

\section{Tala Ilegal (2013) et Umbral (2015) à contre-courant de la photographie du monde urbain amazonien}

5 Parmi les expositions amazoniennes des dernières années, qu'elles soient d'art plastique, de photographie, ou qu'elles regroupent tous les types de média, nombreuses sont celles qui se centrent sur un aspect de la vie urbaine amazonienne, celui de la fête et de la nuit. Les éléments que l'on retrouve dans la plupart des œuvres montrées sont :

les couleurs vibrantes, la sexualité débordante et explicite, des allusions constantes aux effets hallucinogènes de l'ayahuasca (un breuvage chamanique aujourd'hui popularisé) très lié à la vie urbaine d'Iquitos, la capitale du département du Loreto, qui compte plus de 400000 habitants. Un art populaire d'origine spontanée qui pourrait se penser comme du naïf-sauvage. (Jarque F., 2011)

Si le travail conceptuel de l'ensemble de ces expositions était nécessaire car il a permis de déconstruire le lieu commun de la forêt comme lieu de la barbarie ou de frontière avec la polis, elles ont également contribué à associer la représentation de l'Amazonie à une esthétique "criarde» dite tropicale. Dans ce panorama, le choix de sujets photographiques de Musuk Nolte ayant trait à un paysage urbain amazonien détonne. Sa participation lors de la biennale de photo de Lima de 2012 à l'exposition « Selva Virgen, Salvaje y Sensual » («Forêt vierge, sauvage et sensuelle ») nous en offre un exemple. Pour cette exposition de photographies représentant la sensualité habituellement attribuée aux habitants de l'Amazonie et notamment aux résidents d'Iquitos, les photographies de Musuk Nolte retenues proviennent d'un reportage réalisé avec son collectif photojournalistique VersusPhoto sur le CREMI, un hôpital psychiatrique à Iquitos. Outre le noir et blanc, qui contraste avec presque toutes les autres photographies exposées, telles que celles d'Adrián Portugal ou de Morfi Jiménez, la série de Nolte n'a rien à voir avec l'imaginaire populaire au Pérou présent dans l'exposition qui consiste à penser les Amazoniens - et notamment les femmes - comme des gens à la sexualité débridée du fait du climat chaud et humide ${ }^{7}$. Les photographies de Nolte représentent au contraire la fraîcheur avec des portraits de patients du CREMI se baignant dans l'eau mais dont on ne voit que les éclaboussures sur leur visages et corps. L'élément aquatique est d'ailleurs un des seuls éléments caractéristiques des séries amazoniennes de Musuk Nolte, synecdoque 
des grands fleuves de la région et de la chaleur et unique marqueur de cette réalité géographique dans la série. Le climat tropical ne se retrouve donc que par allusion ici. Plutôt que d'être un trait essentialiste des Amazoniens qui expliquerait leur hypersexualisation, elle n'est finalement que signe de l'espace où se situe le sujet photographié.

7 Outre son inclusion quelque peu décalée dans ces expositions collectives, que l'on peut expliquer par le caractère assez réduit du circuit artistique péruvien, d'autres séries amazoniennes de Musuk Nolte révèlent en creux des images stéréotypées auxquelles cette nouvelle vague de représentation visuelle de l'Amazonie ne s'était pas attaquée. Un bon exemple en est son reportage Umbral («Seuil») sur le même hôpital psychiatrique d'Iquitos mais cette fois-ci réalisé seul et dans le cadre de la master class du World Press Photo de 2015 qui avait pour thématique "l'invisible». Cette série de quatorze photographies prises de nuit pour la plupart, en noir et blanc, alterne des portraits flous des patients avec des images de la nature environnante ou d'objets énigmatiques comme autant de fragments de ce que Nolte imagine être la perception qu'ont les malades de la réalité. L'association d'une condition clinique somme toute assez banale - la condition psychiatrique - à l'environnement amazonien produit un dialogue inhabituel dans la mesure où il brise le fantasme des tropiques générant leurs propres virus. Umbral peut s'interpréter alors comme une rupture du déterminisme géographique construit depuis une Europe coloniale dans un but de domination, comme l'a étudié l'historien James Morris Blaut qui s'est basé sur l'exemple des jungles asiatiques de l'empire britannique (Blaut JM., 1996). Point de fièvre jaune, de malaria ou de lèpre dans la série de Musuk Nolte, mais la folie et, qui plus est, celle des habitants d'Iquitos et non celle de l'homme blanc qui perd la tête dans une jungle qui lui est hostile, lieu commun abondamment exploité en littérature ${ }^{8}$. Umbral a pour objectif d'illustrer ce qu'un autre état de conscience permet de voir d'un espace aussi particulier que la forêt.

8 À propos de cette série, Nolte déclare que l'objectif n'était pas tant de documenter la condition mentale des patients que de représenter

cette relation [avec la nature] du patient se trouvant dans un état supérieur de conscience ce qui peut paraître contradictoire car il s'agit d'une liberté conditionnée par la maladie. Ce qui était intéressant c'est cette sorte d'exercice d'un excès de liberté par rapport à l'espace de la forêt ${ }^{9}$.

Il est sans doute malhabile de parler de liberté d'un malade psychiatrique tout en s'attribuant son regard. Il n'est pas question dans cette série de donner la possibilité aux patients de s'exprimer au moyen de l'outil photographique, expérience pourtant classique dans les hôpitaux psychiatriques. Cette position problématique du photographe est néanmoins pleinement assumée, comme l'indique la citation qui accompagne la série : «Seul le frisson initial de mon origine restera à frémir sur le papier noir? ». Ce vers, du poète péruvien José Watanabe et extrait du poème «La photographie", évoque la préoccupation $\mathrm{du}$ poète à l'idée de se faire révéler dans la chambre noire d'un photographe qui lui a tiré son portrait. C'est donc sous l'égide du photographié et non du photographe que Nolte place Umbral. Si cette appropriation du regard d'autrui peut être questionnée, ces clichés rendent visibles des laissés-pour-compte de la société péruvienne et des expositions collectives sur l'Amazonie tout en fournissant, par la double médiation de la photographie et de la perception volée, un nouveau mode de représentation de la forêt. Ce que Nolte appelle le niveau supérieur de conscience passe dans cette série par une délégation de la lucidité du regard aux patients. Les portraits qu'il fait de ces hommes 
et qui semblent correspondre à la vision du photographe sont soit flous (fig.1), soit plongés dans l'ombre, soit voilés. Au contraire, les clichés de paysage qui sont donc posés comme la reconstruction que Nolte fait de la perception des patients, sont plus nets, avec une plus grande profondeur de champ et un éclairage qui fait ressortir les arbres de leur environnement (fig. 2).

Fig. 1 : Umbral (2015), portrait d'un patient de l'hôpital psychiatrique du CREMI.

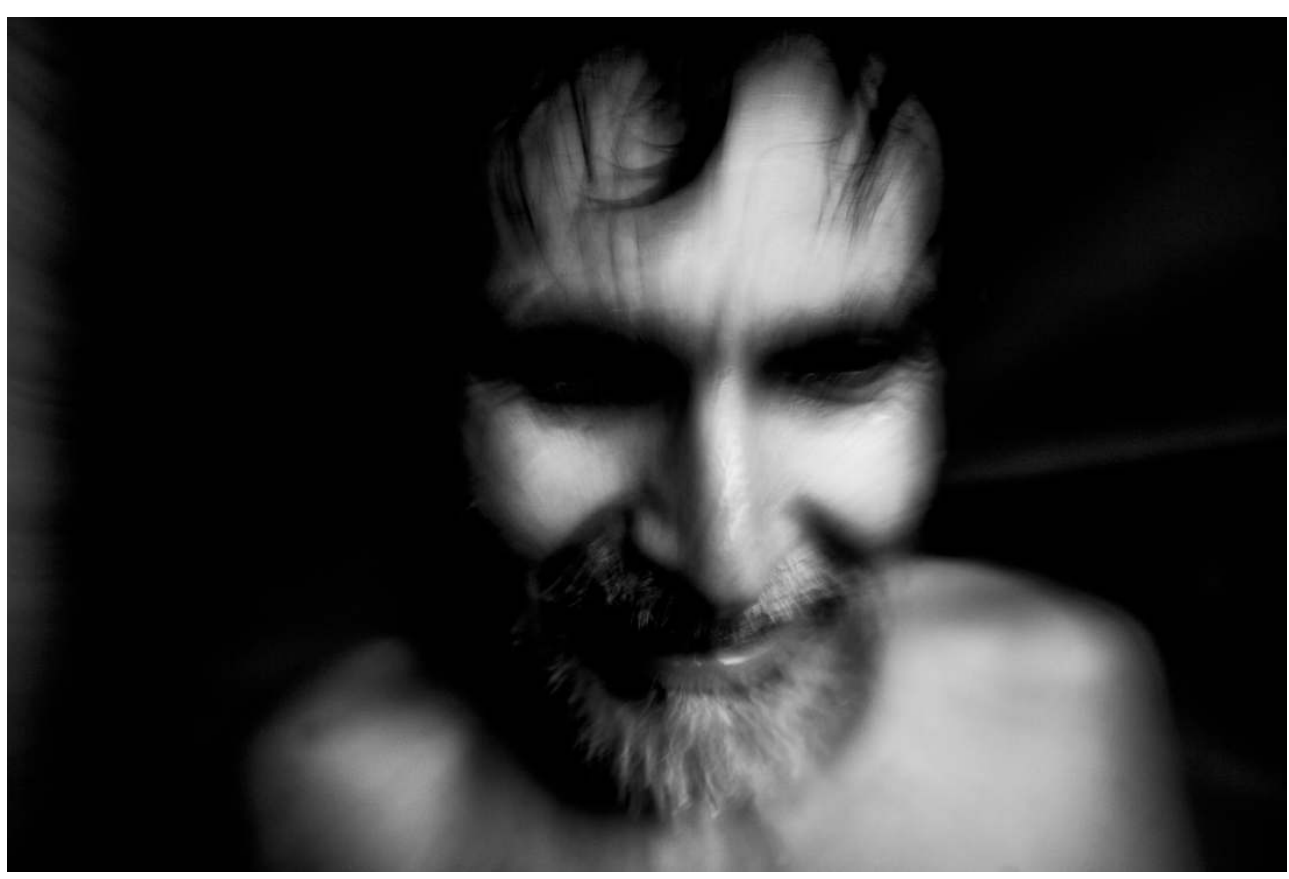

(c) Musuk Nolte (reproduit avec l'aimable autorisation du photographe).

Fig. 2 : Umbral (2015), paysage environnant le CREMI, pris de nuit.

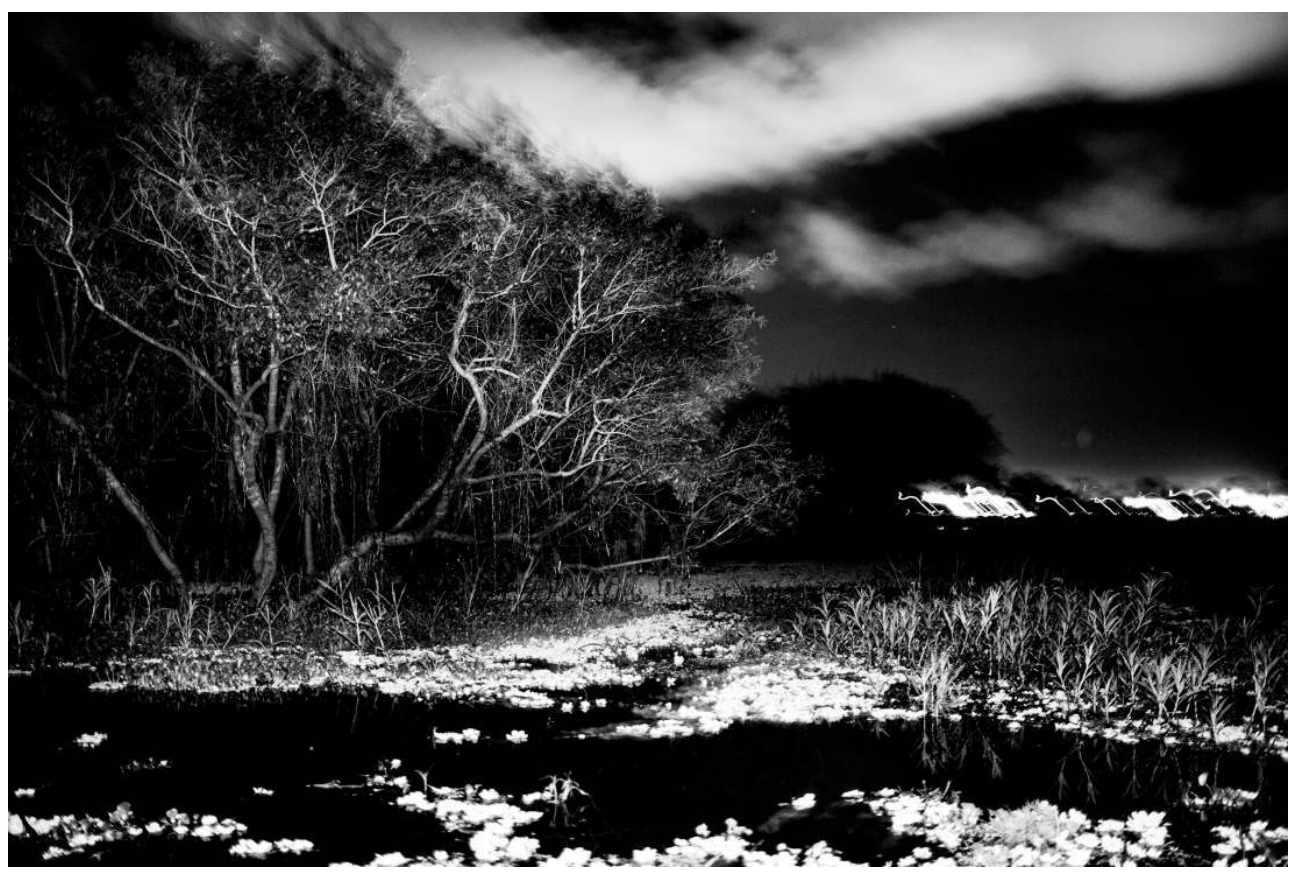

(c) Musuk Nolte (reproduit avec l'aimable autorisation du photographe). 

photographe qui revendique «un regard depuis la pénombre» (Nolte M., 2015a) et la vision paradoxalement plus lucide des patients. Umbral devient la mise en scène de l'impossibilité de Nolte de cerner son sujet qui lui, au contraire, éclaire par son regard la forêt: le «seuil» est alors cet entre-deux, entre deux états de conscience, entre la lumière et l'ombre. Le photographe qui devait répondre au thème imposé de «l'invisible » relève ainsi le défi en imaginant une perception inconnue et justement invisibilisée d'un espace pourtant très photographié.

11 Si la plupart des séries documentaires de Musuk Nolte ont été exposés, la série Tala Ilegal ("Exploitation forestière illégale»), qui aborde un autre aspect du monde urbain amazonien, a été très peu divulguée. Deux des clichés de la série ont fait partie de l'exposition collective de 2013 "Madre Tierra: el lente con el que miramos nuestro planeta » («Terre-mère : l'objectif par lequel on regarde notre planète »). L'exposition, paradoxalement organisée par la compagnie pétrolière nationale PetroPerú, proposait de réunir onze photographes autour de la question de l'engagement écologique et la destruction de la planète. Hormis cette exception, Tala Ilegal n'a circulé que dans le cadre d'une enquête journalistique de la revue Poder sur le trafic illégal de bois dans le département de l'Ucayali (Acosta C., 2014), alors que les clichés ont été pris dans le port fluvial de Masusa, à Iquitos, dans le département du Loreto. Dans les deux cas, remarquons que le propos initial de la série est dévié, que ce soit par un déplacement géographique ou par un déplacement de sens. En effet, l'exposition "Madre Tierra " réunissait principalement des photographies de paysages somptueux à préserver plutôt que des processus de destruction de ceux-ci. Le sujet même de la série documentaire de Nolte et le parti pris choisi sont des éléments qui peuvent expliquer sa circulation limitée ou détournée. Depuis une perspective écologique, les photographies de la déforestation sont habituellement perçues sous l'angle de la forêt comme victime ${ }^{10}$ ou du point de vue de dirigeants indigènes défendant leur droit à disposer de leur terre. Pour citer un exemple, le dirigeant de la nation ashaninka, Edwin Chota, assassiné en 2014 justement car il s'opposait aux trafiquants de bois en Amazonie péruvienne, a été immortalisé dans sa lutte par plusieurs photographes, comme Tomás Munita pour le New York Times. Au contraire, Tala Ilegal rappelle une tradition pré-écologique de représenter l'industrie de la coupe de bois comme source de développement pour le pays, tout en faisant des travailleurs du bois les personnages principaux de la série documentaire. Ces hommes, derniers maillons d'un trafic qui est certes posé comme «illégal» mais dont ils ne perçoivent qu'à peine les bénéfices, sont présentés non comme une menace allogène au monde de la forêt mais comme faisant partie à part entière de la réalité amazonienne. Musuk Nolte se détache ainsi d'une représentation type de l'Amazonie que l'anthropologue Stephen Nugent décrit en ces termes:

Les maux [qui frappent la forêt] ne peuvent être niés, et pourtant ils ne sont généralement pas présentés comme partie intégrale de l'Amazonie mais comme des facteurs de perturbations qui peuvent être atténués («arrêtez d'abattre des arbres », "préservez la biodiversité »), conservant ainsi la séparation conventionnelle entre systèmes biologiques et systèmes sociaux tout en niant la longue histoire humaine de la modification du terrain. (Nugent S., 2007 :47)

Dans la série de Nolte, c'est l'ensemble de l'économie du trafic du bois que l'on retrouve, depuis la taille effective, jusqu'à la transformation du tronc en planche de bois. Ces travailleurs, qui sont aussi des habitants des villes amazoniennes auraient toute leur place dans la représentation de l'urbanité. La série repose à la fois sur une 
suresthétisation de la déforestation en soi et sur le travail documentaire sur les conditions de vie de cette main d'œuvre informelle. Elle apparait donc incompatible avec les émotions habituellement sollicitées d'un public confronté aux images de destruction de la nature. Dans Tala ilegal, on trouve des clichés où la beauté de l'amoncellement de troncs coupés, le noir et blanc extrêmement contrasté annulent immédiatement l'association des troncs rouges à une violence qui leur serait faite et les dissocie du plus grand ensemble d'où ils proviennent, la jungle tropicale et verte (fig. 3). Ils constituent une nouvelle unité à part entière, matière brute tronçonnée et prête à usage : ce n'est plus une béance dans une vue du ciel de la forêt, mais un relief.

Fig. 3 : Tala llegal (2013), jeune garçon assis sur un amoncellement de troncs coupés.

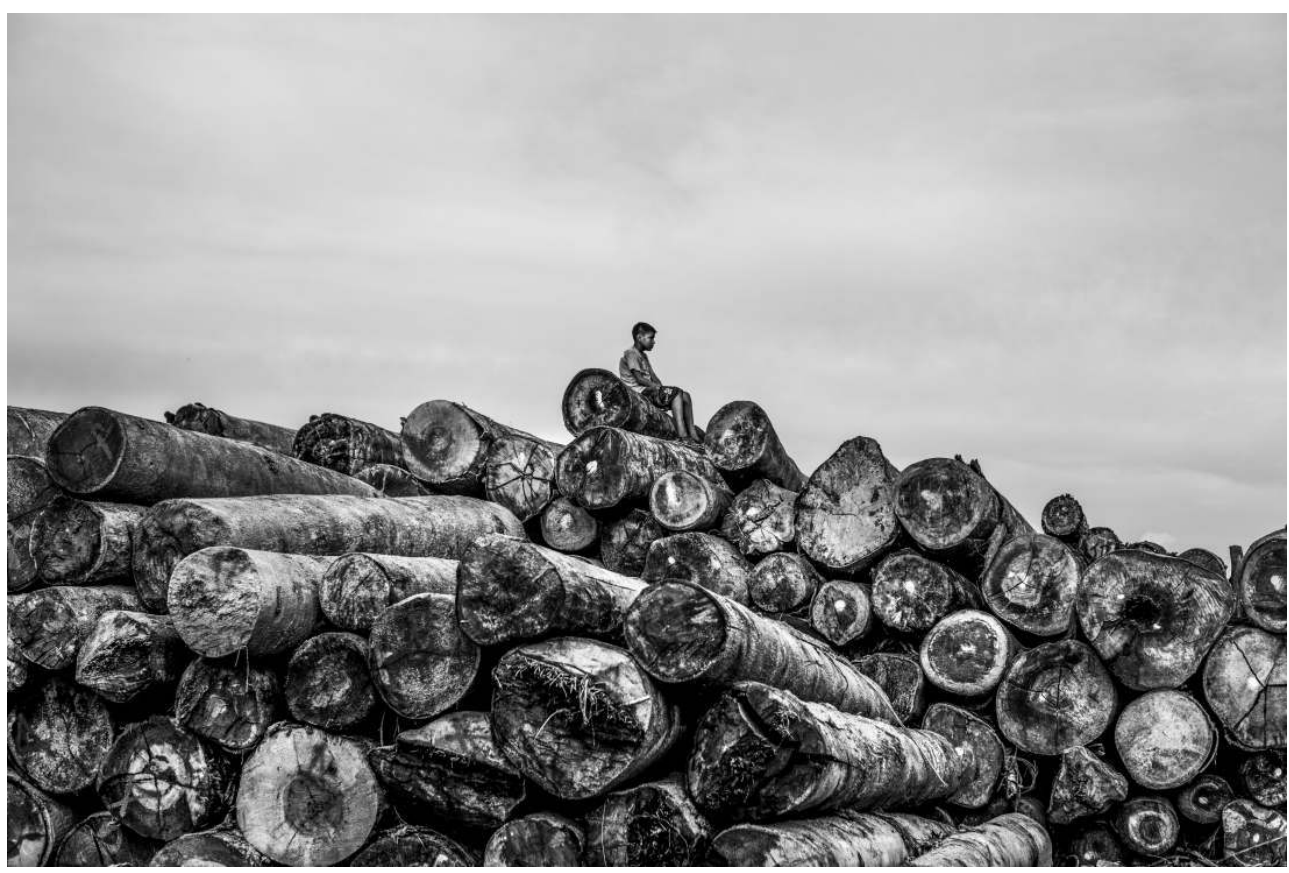

(c) Musuk Nolte (reproduit avec l'aimable autorisation du photographe).

Dans cette photographie, un enfant spectateur du trafic est assis sur une montagne d'arbres et devient d'autant plus minuscule que l'empilement est important. La déforestation crée alors un nouveau paysage, un nouveau terrain quelque peu escarpé qui divise le cliché en deux parties égales, entre les troncs et le ciel. Tous les travailleurs de cette série sont d'ailleurs autant de corps souffrants, écrasés par le poids des troncs disproportionnés ${ }^{11}$. Face à une répétition allant jusqu'à une impression d'infini des arbres abattus, face à cette invariance, Nolte photographie surtout des travailleurs seuls ou en binôme, isolés face à la pénibilité de la tâche (fig. 4). 
Fig. 4 : Tala llegal (2013), un travailleur au milieu des troncs coupés.

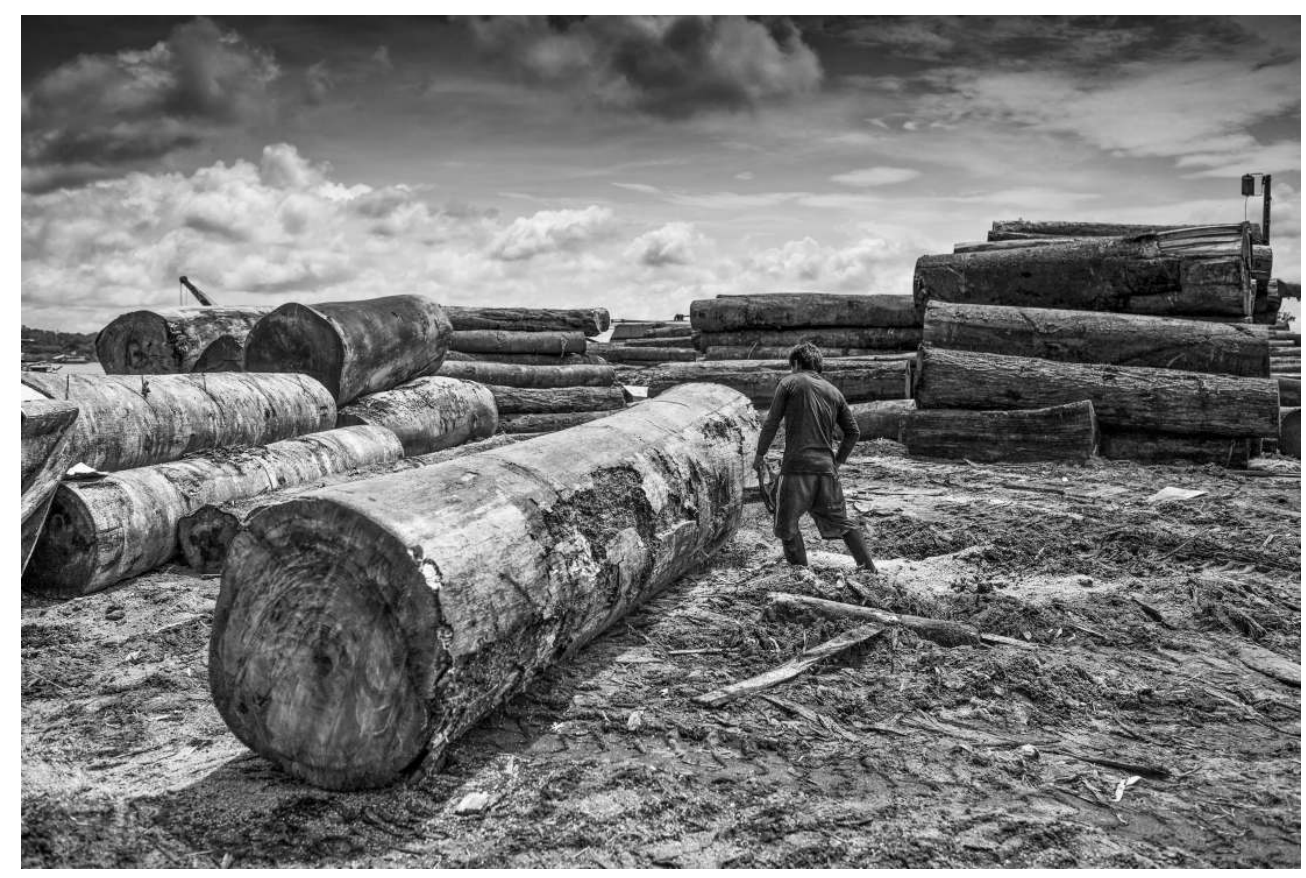

(c) Musuk Nolte (reproduit avec l'aimable autorisation du photographe).

Un cliché isole un travailleur dans un champ de troncs lui arrivant à hauteur de buste. Le personnage légèrement décalé sur la droite du centre du cadre cède tout le premier rôle à la diagonale du tronc. Celui-ci, qui par sa taille monumentale semble impossible à maîtriser, devient alors l'élément hostile pour le travailleur dont le rôle d'agresseur de la nature disparaît. Le paysage entier est un paysage de troncs que l'on retrouve à tous les plans, disposés de façon diversifiée et encerclant l'homme dont l'espace de circulation est entièrement réduit au quart du cliché. Seule la grue que l'on aperçoit à l'arrière-plan rappelle tout l'appareillage technique nécessaire à une entreprise de cette ampleur. La suite des clichés de la série s'arrête à toutes les étapes de la découpe du bois, de la scierie jusqu'au transport final de planches. Dans un dernier cliché, nous retrouvons les travailleurs ployant désormais sous le poids de planchettes ridiculement fines en comparaison avec la matière brute. Tel est l'autre pan de la réalité urbaine en Amazonie que photographie Nolte : non pas à la nuit tombée au sein de la fête psychédélique et tropicale, mais dans ce que l'existence de ces villes au milieu de la forêt et leur subsistance implique - une économie principalement basée sur le trafic, ici de bois, dans d'autres cas de l'exploitation minière illégale. Ainsi, les sujets ayant trait au monde urbain amazonien retenus pour faire l'objet de documentaires photographiques par Musuk Nolte sont à contrecourant des thématiques des expositions collectives des dernières années. Leur circulation est alors souvent déviée de leur propos initial. Finalement, ce sont surtout ses séries sur des peuples indigènes d'Amazonie, notamment celle sur les Shawis, qui se retrouvent au cœur du circuit d'exposition de Lima mais aussi de la présence de la culture péruvienne à l'étranger. Shawi présente un dernier aspect de la photographie à thématique amazonienne de Nolte: une stratégie d'évitement de la photographie ethnographique et de l'exotisme. 


\section{Shawi (2009) ou l'évitement d'une photographie ethnographique et exotique}

Composée de neuf ${ }^{12}$ photographies carrées en noir et blanc, Shawi est la série de Musuk Nolte qui a le plus circulé. Remportant le prix national Eugène Courret en 2010 à Lima, la série a ensuite été exposée, entre autres, à Paris à la biennale Photoquai en 2013, en Equateur pour l'édition de 2015 de Fotoquito, en 2016 à Mexico à la galerie MYL arte contemporáneo et en 2017 à Lima au Centro Cultural Peruano Británico. Interrogé sur la raison du succès de cette série, Nolte suppose qu'elle correspond à ce que l'on attend d'un photographe latino-américain (Nolte, entretien personnel, 18 février 2017). Il est vrai que dans les textes de présentation des expositions comme dans la propre description qu'en fait le photographe, le caractère magique ou onirique de ces clichés est souligné : «Les Shawis sont un mystère par nature. [...] C'est l'ethnie qui a su le mieux préserver son territoire, son langage, sa coexistence millénaire avec les forces mystiques de la forêt à travers leurs mythiques chamanes qui, par leur isolement volontaire, ont toujours été les plus respectés dans les légendes amazoniennes ${ }^{13}$.» Selon Mathieu Corp, ce type de posture correspond typiquement à l'attente du public européen face à la photographie latino-américaine contemporaine : retrouver le réalisme magique si souvent associé au continent, ce qui exotise d'autant plus les sujets photographiés (Corp, 2015 : p. 122-125). S'il est vrai que les effets stylistiques de la série de Nolte transmettent cette impression d'onirisme, s'opère également un refus d'exotisme. Nous entendons ce mot dans le sens que lui donne Peter Mason :

L'exotique est produit par un processus de décontextualisation: pris d'un environnement venant d'ailleurs (c'est cet ailleurs qui le rend exotique), il est transféré dans un environnement différent ou recontextualisé. $\mathrm{Ne}$ sont pas valorisés les contextes géographiques et culturels "originaux ", mais la capacité des objets en question de prendre un nouveau sens dans un nouveau contexte.

(Mason P., 1998 : 3)

L'exotique est alors cette altérité que l'on rend familière en la rapprochant d'un contexte connu. Afin de comprendre en quoi Shawi peut s'interpréter comme un refus d'exotisme, un retour sur la genèse de la série est nécessaire. Suite à une commande du quotidien $\mathrm{El}$ Comercio sur la résistance de la langue de la nation Shawi dans le Haut Amazone péruvien, paraît le 14 septembre 2009 un article de Ricardo León intitulé «Ethnie qui se récupère elle-même » accompagné de six clichés de Nolte. Ce reportage devient une occasion pour le photographe de réaliser son propre projet. Les clichés publiés dans le journal n'auront rien à voir avec ceux retenus pour sa série personnelle ${ }^{14}$. En effet, la plus grande des photographies du reportage publié dans El Comercio relève d'un type de procédé d'exotisation (fig. 5). 
Fig. 5 : $S / T$ (2009), portrait d'une jeune fille shawi portant les peintures de visage réservées aux occasions spéciales.

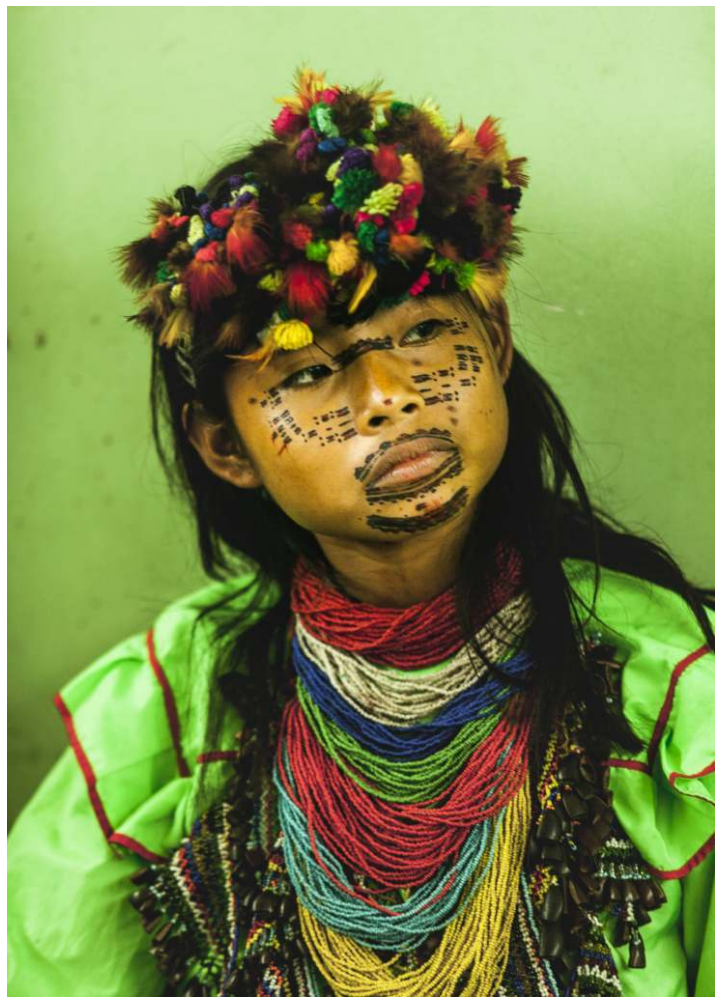

(c) Musuk Nolte (reproduit avec l'aimable autorisation du photographe).

Sur ce cliché, une jeune fille shawi, portant toutes les parures qui permettent de l'identifier ethniquement et de la fixer dans son altérité, sur un fond uni gommant tout contexte, est offerte au lecteur comme venant de loin tel que l'indique la légende qui accompagnait originellement la photographie : «Une carte postale » (León, 2009 :16). Le cliché qui n'est pas sans rappeler une esthétique National Geographic, est lié par la légende qui l'accompagne à la mode du «goût de l'autre» datant de la période de grande circulation de cartes postales représentant des communautés indigènes mises en scène à la fin du XIx et au début du xx siècle. La suite de la légende, « les visages peints révèlent un moment: l'arrivée d'un invité, les adieux, une célébration; c'est un trait typique shawi»(ibid.), renoue avec une tradition de photographie anthropologique de classification de « types » humains.

À l'inverse, dans sa série personnelle, Nolte gomme le plus d'informations ethnographiques possible, annulant l'effet d'altérité propre à l'exotisme et parlant d'ailleurs de «malaise» quand il s'agit des clichés pour El Comercio à cause de l'objectivation qu'ils impliquent (Nolte M., entretien personnel, 18 février 2017). Lors de l'exposition de Photoquai, deux photographies de jeunes femmes portant ornements et maquillage traditionnels qui ne se trouvent pas dans la série initiale telle que Nolte la présente sur son site ont été rajoutées, comme s'il s'agissait de répondre aux attentes d'un public d'une biennale dont le sous-titre est « images du monde ». Mais dans la série originale, aucune information photographique n'est donnée permettant d'identifier les Shawis, hormis le nom de cette nation qui est le titre de la série. Cette affirmation souligne d'ailleurs un abus historique, les clichés de peuples indigènes ont souvent fait 
l'objet de confusions ethniques, une image valant pour une autre, un peuple pour un autre, dans une interchangeabilité de l'altérité (La Serna J.C., et Biffi V., 2017). Nolte gomme beaucoup d'informations contextuelles, non pas pour décontextualiser puis recontextualiser comme dans une recherche d'exotisme, mais pour non contextualiser et ainsi atténuer ce qui évoque l'extrême altérité. Ses clichés ne montrent le cadre de la forêt amazonienne que sous la forme de fragments avec des éléments métonymiques de cet espace, comme l'eau. L'aspect fragmentaire est d'ailleurs souligné par le format carré des tirages, unique dans la pratique de Nolte, et qui sont comme autant de découpes dans l'espace et dans la situation qu'il documente. Le carré est également une façon d'éviter tout cadrage plus spectaculaire, se limitant ainsi au détail pour souligner la recherche du quotidien et non de l'exceptionnel. La photographie du chaman (fig. 6) est symptomatique de l'effacement du contexte.

Fig. 6 : Shawi (2009), portrait d'un chaman shawi.

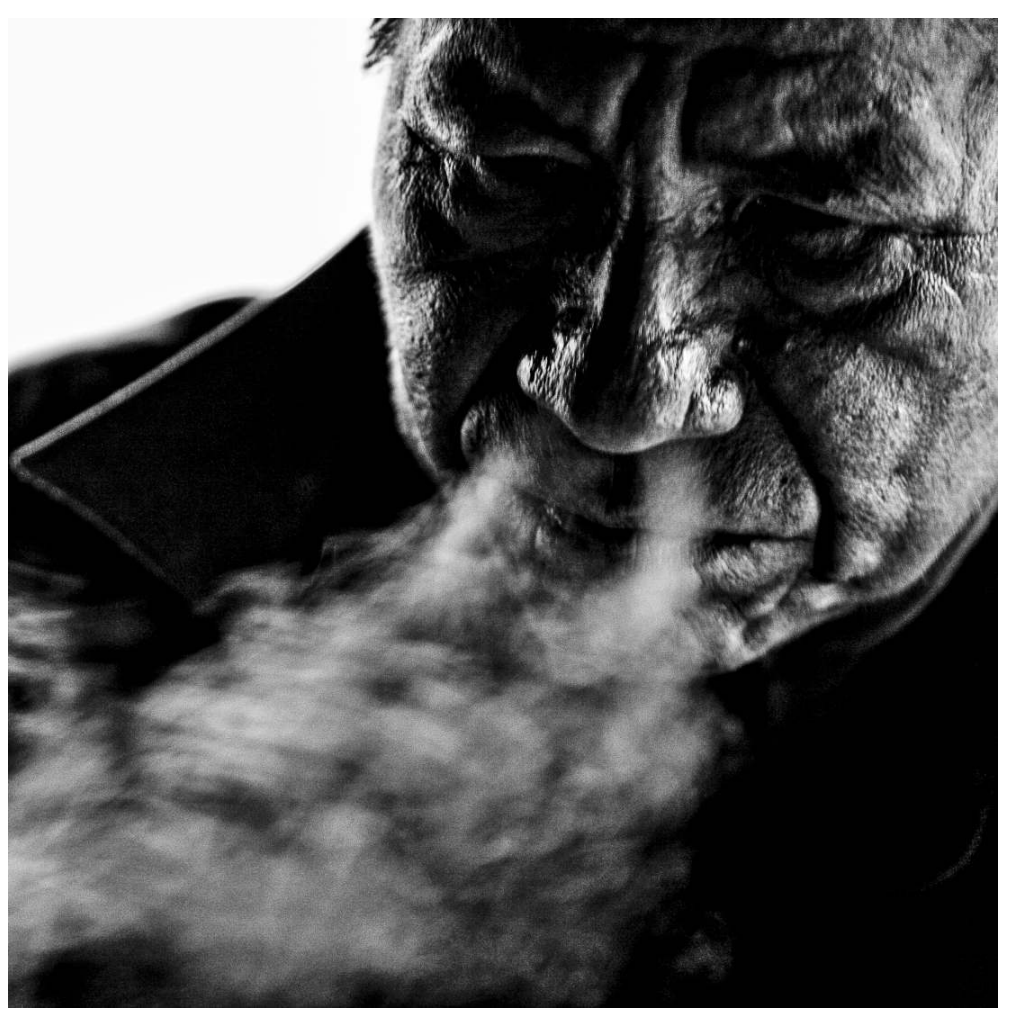

(c) Musuk Nolte (reproduit avec l'aimable autorisation du photographe).

19 Hormis la fumée provenant probablement d'un mapacho, le cigare rituel amazonien qui indiquerait la fonction chamanique du personnage, aucune autre information ethnographique ne peut être tirée du cliché, ce n'est d'ailleurs que lors d'entretiens que Musuk Nolte précise la fonction de l'homme photographié. Le plan rapproché sur le visage place dans le hors champ toute information du contexte et l'arrière-plan demeure blanc. Pourtant, cette photographie, si énigmatique quand elle est isolée du reste de la série, est celle qui a le plus souvent illustré les articles de presse parlant des expositions collectives de photographie amazonienne. Cette limitation d'information contextuelle est également frappante dans une photographie du chef de la communauté shawi de Fray Martín (fig. 7), fonction qui, une fois encore, n'est révélée que dans les entretiens de Nolte : 
Fig. 7 : Shawi (2009), portrait de Robinson Pinedo, chef de la communauté de Fray Martín.

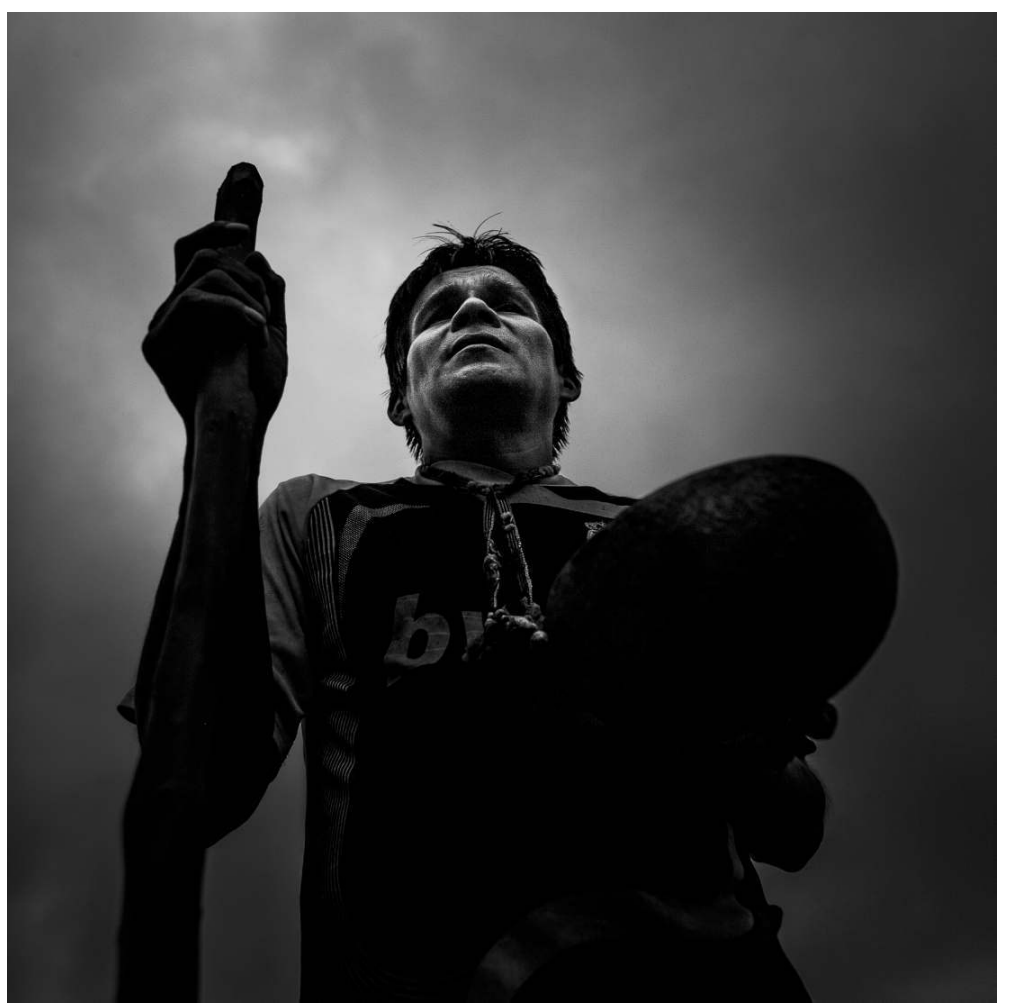

(c) Musuk Nolte (reproduit avec l'aimable autorisation du photographe).

Si la contreplongée, le bâton et l'illumination qui auréole la tête de l'homme indiquent sa position de dirigeant, aucun autre élément ne permet de situer cet homme qui, d'ailleurs, porte un maillot de football très facilement reconnaissable. Ces deux portraits nient d'office le «mythe des "peuples premiers" incarnant une forme contemporaine du "goût des Autres” » (De l'Estoile B., 2012 : 364), selon l'expression de Benoît de l'Estoile au sujet, entre autres, des photographies de Sebastião Salgado sur les Xingu de l'Amazonie brésilienne. Chez Nolte, le refus de l'énonciation d'information anthropologique est un refus d'esprit de classification et d'analyse scientifique de ces populations, notamment dans le contexte péruvien déjà évoqué de redécouverte de la photographie de missions de la fin du XIX ${ }^{e}$ siècle et de ses abus. Comme le dit Alejandro Castellote à propos du livre Piruw édité par Musuk Nolte où l'on retrouve les photographies de Shawi :

Dans la photographie anthropologique $\mathrm{du} \mathrm{XIX}^{\mathrm{e}}$, nommer c'est représenter, présenter aux Européens des cultures différentes comme autres. L'influence de ce paradigme colonial et ethnocentrique est telle dans notre imaginaire visuel, qu'il est courant de voir des photographes asiatiques, africains ou latino-américains enregistrer leurs gens comme s'ils étaient des apprentis anthropologues du XIX ${ }^{\mathrm{e}}$. C'est pour cela que l'inexactitude scientifique de ce travail sur le Pérou est une bonne surprise [...]. C'est une histoire à la première personne qui n'est, ni de l'intérieur, ni de l'extérieur. (Castellote, 2014)

La question de cette première personne est centrale dans le projet Shawi. Comme l'écrit Diego Lizarazo Arias dans le texte de présentation de l'exposition de Nolte à la galerie mexicaine MYL :

Que la presse ait défini comme « reportage » le travail qu'a élaboré Musuk avec les Shawis est un peu trompeur. L'expérience qui affleure et que les images 
contiennent est plus proche d'une exploration et d'un voyage en quête de connaissance, au sens existentiel et indigène de ces concepts. (Lizarazo Arias D., 2016)

Nolte souligne dans plusieurs entretiens qu'il a profité de son éloignement et isolement géographique - les Shawis vivant à deux jours de navigation de la ville la plus proche du Haut Amazone - pour se trouver dans un état de perception nouveau. Selon lui, « être loin de chez toi, émotionnellement, ça te prédispose à voir les choses autrement » (Nolte, M., 2015). Dans cette série, ce ne sont pas les Shawis qui sont montrés comme un peuple isolé, peuple premier plus ou moins préservé de la modernité, comme dans beaucoup de photographies de communautés indigènes, c'est Musuk Nolte qui montre sa propre sortie d'une zone de confort. Néanmoins, ce qu'il documente n'est pas l'inadaptation d'un homme blanc dans la jungle, mais une façon de voir qui serait nouvelle, une façon de photographier différemment. Venant pour faire un reportage sur les stratégies de résistance culturelle de la communauté, Nolte va surtout découvrir l'importance des chamans shawis et finir par documenter cet aspect-là de la communauté. Ayant participé à une cérémonie d'ayahuasca, mélange de plantes psychotropes utilisé dans quasiment tous les rituels chamaniques amazoniens, Nolte dit s'être inspiré de cet état de conscience pour prendre ses clichés (ibid.). Néanmoins, il ne s'agit pas d'un reportage fait dans un état de transe ou d'un travail documentaire du rituel en soi, type de photographies dont on peut trouver des exemples chez le photographe péruvien Daniel Silva. Il s'agit plutôt, une fois le rituel et la prise d'ayahuasca finis, de capturer des visions qui auraient adhéré au réel et qui seraient comme autant de référents à photographier. Selon ses propres mots, il était question « de choisir certaines images par l'imaginaire qu'[il avait] reçu de l'ayahuasca " (ibid.). Nolte parle souvent du territoire amazonien comme composé de couches de réalité à révéler. Tout comme dans la série Umbral où il tentait de s'approprier un autre état de conscience pour dévoiler ces couches cachées, il profite de la mémoire des effets de l'ayahuasca pour percevoir autrement ses sujets. Les neuf clichés documentent alors deux éléments. Tout d'abord, le quotidien des Shawis comme les scènes de pêche la nuit ou les moments en famille autour du feu, mais également l'aspect latent du poids des chamans dans ce quotidien. Cette autre couche de vérité, Nolte la retrouve aussi par son hyper contrôle de l'éclairage: comme souvent, il préfère photographier la nuit, seule façon pour lui de maîtriser la source de lumière. L'autre état de conscience tiré de l'expérience de l'ayahuasca permet de photographier en adoptant un nouveau regard, quasiment celui d'une bête qui chasse ${ }^{15}$, avec des prises au ras du sol ou en contreplongée. Cette vision de prédateur est par exemple présente dans les clichés où les hommes sont des silhouettes lointaines (fig. 8). 
Fig. 8 : Shawi (2009).

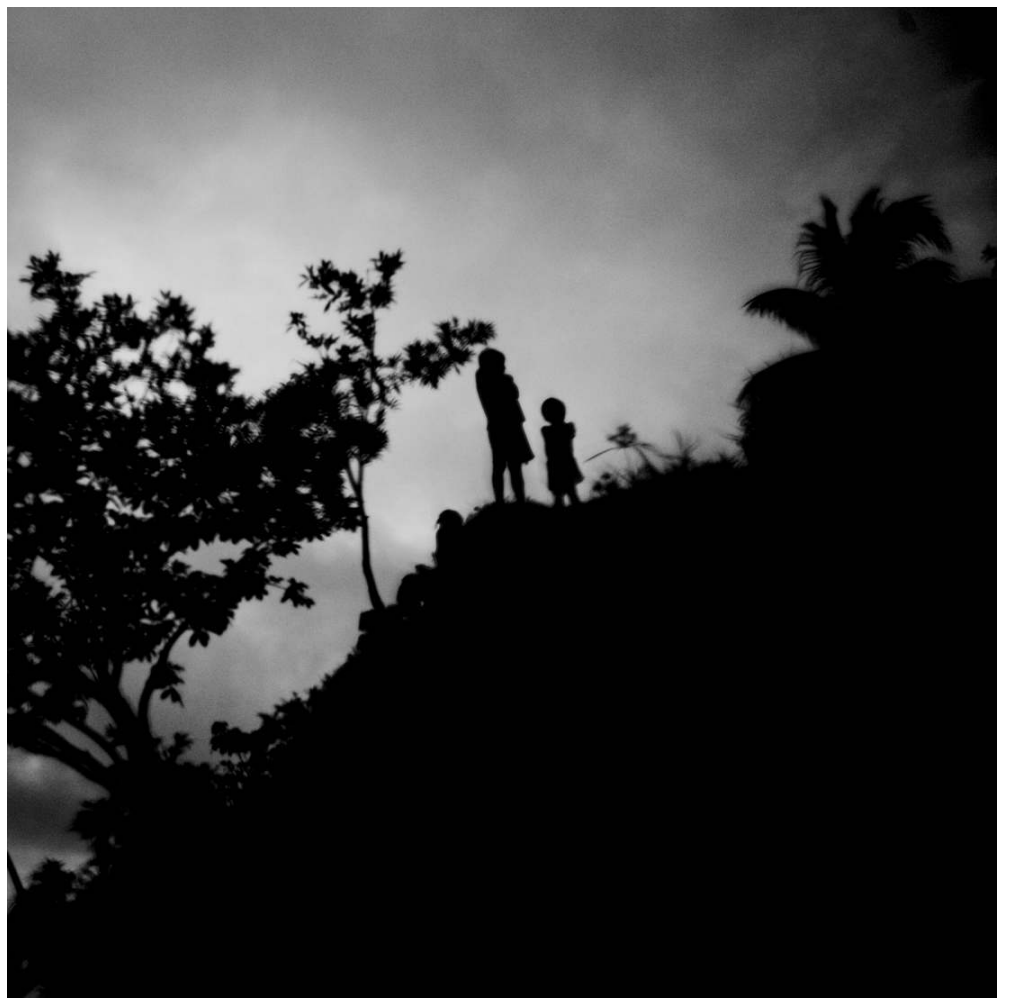

(c) Musuk Nolte (reproduit avec l'aimable autorisation du photographe).

C'est ce même regard que l'on retrouve dans le portrait d'un homme shawi (fig. 9) où le jeu de lumières évoque un feuillage dans lequel serait tapi le photographe. 
Fig. 9 : Shawi (2009).

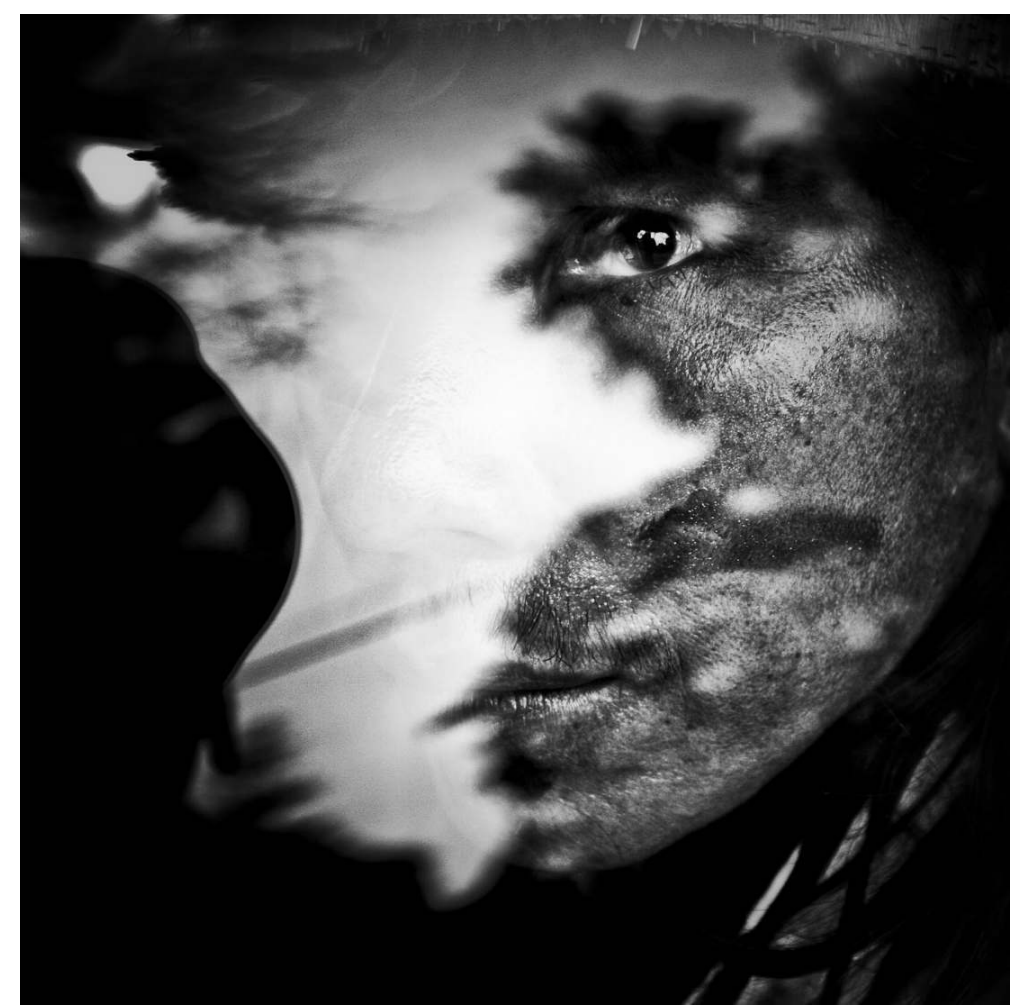

(c) Musuk Nolte (reproduit avec l'aimable autorisation du photographe).

Paradoxalement caché parce que trop éclairé, l'homme shawi semble également porter un regard de chasseur sur une proie, regardant et regardé se confondant dès lors dans cette moitié de visage qui appelle à être complétée par celui qui scrute ${ }^{16}$. Ainsi, même si la série n'intègre pas de clichés représentant l'aspect plus urbanisé de la vie de la communauté, ce qui permettrait de nuancer l'aspect magique des photographies, cela n'empêche pas pour autant un travail de refus de représentation du peuple Shawi comme radicalement autre. Contrairement à ce qui s'opère dans une photographie qui cultive le "goût de l'autre " et qui, selon Benoît de l'Estoile, présente la part perdue de nousmêmes, ce cliché de Musuk Nolte supprime les codes habituels de l'altérité et suspend toute quête d'un ailleurs.

\section{Conclusion}

Le travail du photographe Musuk Nolte fait partie de la revalorisation actuelle des représentations visuelles de l'Amazonie au Pérou. Qu'il s'agisse des trois séries étudiées ici - Shawi, Tala Ilegal et Umbral - ou de ses autres travaux réalisés dans la forêt amazonienne comme Asháninka ou Bosque anfibio, Nolte participe de l'«économie visuelle » de cet espace, pour reprendre le concept de Deborah Poole. Si chaque série prise individuellement peut tomber dans certains écueils, elles contribuent dans leur ensemble à déconstruire d'autres stéréotypes.

Interrogé sur sa relation à l'espace amazonien tel qu'il revient dans ses séries photographiques, Musuk Nolte parle d'une Amazonie «non pas dans le sens géographique, mais comme construction symbolique ${ }^{17} »$. Dans presque toutes ses 
interviews reviennent deux idées : celle de se situer dans un entre-deux et celle de révéler des couches de la réalité. Le style artistique de ses documentaires est finalement là pour assurer la révélation de qui est caché. D'ailleurs, s'il est aujourd'hui connu pour ses séries amazoniennes, la forêt est très absente de ses séries, elle est hôpital psychiatrique, elle est déforestation et les Shawis ne portent aucun attribut qui permette de les identifier ethniquement comme peuple amazonien. Tout comme le symbole dans son sens propre comme figuré résulte de l'union de deux parties pour faire sens, les séries amazoniennes de Musuk Nolte résultent de la réunion du style artistique et de son intention documentaire, seul moyen pour lui d'accéder aux couches de l'invisible.

\section{BIBLIOGRAPHIE}

Acosta, Christopher, « Relatos salvajes », Poder, janvier 2014, p. 26-37.

Castellote, Alejandro, " On Musuk Nolte and Leaslie Searles's Piruw », Emaho Magazine, 24 avril 2014, http://www.emahomagazine.com/alejandro-castellote-on-musuk-nolte-and-leslie-searlesspiruw/, page consultée le 21 novembre 2017.

Corp, Mathieu, Des expériences du temps dans la photographie latino-américaine contemporaine, Thèse d'art et histoire de l'art, Université Sorbonne Nouvelle-Paris 3, 2015, https://tel.archivesouvertes.fr/tel-01486997, page consultée le 21 novembre 2017.

De l'Estoile, Benoît, «Images des paradis perdus : Mythe des "peuples premiers”, photographie et anthropologie ", Vibrant - Virtual Brazilian Anthropology, vol. 9, n 2, juillet-décembre 2012, http:// www.vibrant.org.br/issues/v9n2/benoit-de-lestoile-images-des-paradis-perdus/, page consultée le 21 novembre 2017 .

Jarque, Fietta, « Pop lujurioso y geometrías místicas », El País, 20 août 2011, https://elpais.com/ diario/2011/08/20/babelia/1313799180_850215.html, page consultée le 5 juin 2018.

La Serna, Juan Carlos et Biffi, Valeria, « Otredad y permanencia : Los usos de la fotografía histórica en la exposición contemporánea del indígena y el bosque amazónico. ", Revista chilena de antropología visual, $\mathrm{n}^{\circ} 27,2016, \mathrm{p} .119-139$, http:// www.rchav.cl/2016_27_art06_la_serna_\&_biffi.html, page consultée le 28 novembre 2017.

León, Ricardo, « Etnia que se recupera a sí misma », El Comercio, 14 septembre 2009.

Lizarazo Arias, Diego, « Musuk Nolte : El otro nos mira », texte de présentation de l'exposition « El otro nos mira. Vida y espíritu del Amazonas », galerie MYL, Mexico, octobre 2016.

Mason, Peter, Infelicities : Representations of the Exotic, Baltimore, Johns Hopkins University Press, 1998.

Motta, Angélica, « La "charapa ardiente” y la hipersexualización de las mujeres amazónicas en el Perú : perspectivas de mujeres locales ", Sexualidad, Salud y Sociedad, n 9, 2011, p. 29-60, https:// dx.doi.org/10.1590/S1984-64872011000400003, page consultée le 5 juin 2018.

Nolte, Musuk, « Masterclass Latinoamérica 2015 », Zonezerophoto, vidéo d'un entretien de 2015a, https://www.youtube.com/watch ?v =BTt3mSAoszE, page consultée le 28 novembre 2017. 
Nolte, Musuk, « Shawis », $f / 22$. Fotografía en profundiad, vidéo du centre de photographie de Montevideo, 2015b, http://cdf.montevideo.gub.uy/content/musuk-nolte-shawis, page consultée le 28 novembre 2017.

Nugent, Stephen, Scoping the Amazon : image, icon, ethnography, Walnut Creel, Left Coast press, 2007.

\section{NOTES}

1. Le critique d'art et curateur Gustavo Buntinx a par exemple déclaré, dans un cycle de conférences qu'il organisait à Lima du 15 au 17 mai 2017, que le premier grand jalon de l'art péruvien $\mathrm{du} \mathrm{XxI}^{\mathrm{e}}$ siècle était "l'irruption d'une présence amazonienne », notamment depuis la peinture dite chamanique ou visionnaire. Sauf indication contraire, toutes les traductions de l'espagnol vers le français et de l'anglais vers le français ont été réalisées par l'autrice de l'article.

2. Cette affirmation est à nuancer à certains moments de l'histoire du Pérou liés à un contexte précis. Un bon exemple de cela est l'effort déployé par le gouvernement du président Manuel Prado en 1942 pour célébrer le IV centenaire de la découverte de l'Amazone, commémoration qui coïncide avec la fin de la guerre péruviano-équatorienne de 1941 et met l'accent sur la péruvianité de la forêt amazonienne qui se trouvait au cœur dudit conflit.

3. Parmi ces expositions à caractère historique, citons : "L'Amazonie de Charles Kröehle. Photos et cartes postales, fin $\mathrm{XIX}^{\mathrm{e}}$, début $\mathrm{XX}^{\mathrm{e}}$ » (2010), «Images et imaginaires de l'époque du caoutchouc » (2012) et "Photographies de la Commission consulaire au Putumayo et affluents » (2014). Ces expositions ne peuvent être envisagées sans l'important travail de recherche qui les accompagne. Citons, par exemple, l'ouvrage récent sur la photographie historique en Amazonie péruvienne, La Serna Salcedo, Juan Carlos et Chaumeil, Jean-Pierre, El bosque ilustrado: diccionario histórico de la fotografía amazónica peruana (1868-1950), Lima, CAAP, IFEA, PUCP, 2016.

4. L'expression de "réalisme magique » imputable au critique allemand Franz Roh, reprise et diffusée en Amérique latine par l'écrivain vénézuélien Arturo Uslar Pietri et complétée par le concept de « réel merveilleux » de l'écrivain cubain Alejo Carpentier, désigne en premier lieu une esthétique et un projet littéraire révélateur de la cohabitation du merveilleux (ou du magique) et du réel dans l'espace latino-américain. Devenu quasiment une étiquette éditoriale pour les romans latino-américains notamment après le succès de Cent ans de solitude de Gabriel García Márquez, l'expression s'est étendue à d'autres domaines. La coexistence d'éléments décalés, cherchant à émerveiller, au sein de photographies qui prétendent s'ancrer dans une dure réalité sociale latino-américaine, contribuent à pérenniser un goût pour l'exotisme du continent. Dans le cas de la photographie péruvienne contemporaine, citons l'exemple de Javier Silva Meinel.

5. Biographie de Musuk Nolte, http://www.gluciadelapuente.com/artistas/musuk-nolte/, page consultée le 21 novembre 2017. Depuis la rédaction de cet article, cette galerie historique a fermé ses portes.

6. Citons par exemple l'exposition «Fotografía peruana » à la Deutsche Bank de New York en 2013 ou « Perú Amazónico » à Alger et à Sidney en 2013 et au Panama en 2016.

7. Ce lieu commun extrêmement répandu au Pérou, et qu'Angélica Motta désigne par l'expression "charapa ardiente » (Motta A., 2011), peut trouver ses origines dans les premières caractérisations de l'Amazonie comme territoire de la barbarie et du désordre par antonomase lors de la Conquête espagnole. Cette image de la nature indomptable associée à la condition climatique déjà évoquée a donné lieu, par la suite, à la construction des Amazoniens comme des êtres à la sexualité tout aussi indomptable et donc débordante. 
8. Ce topos est très présent dans le genre latino-américain dit de la novela de la selva. On le retrouve également dans d'autres traditions littéraires provenant de contextes géographiques différents tels que la forêt tropicale au Congo dans Au cœur des ténèbres de Joseph Conrad.

9. Cette citation provient de la version non éditée d'un entretien de Musuk Nolte pour Bendayán, Christian (ed.), Amazonistas, Lima, Ministerio de Cultura, Bufeo, 2017.

10. Voir par exemple le reportage d'Alex Webb pour National Geographic en 2013 dans l'Ucayali où la focale est mise sur l'impuissance des pouvoirs publics et des communautés indigènes à lutter contre le trafic de bois. http://ngm.nationalgeographic.com/2013/04/mahogany/webbphotography, page consultée le 27 novembre 2017.

11. L'esthétique de cette série peut rappeler en ce sens celle de Sebastião Salgado dans la Serra Pelada, mine d'or du Brésil qu'il reproduit dans La Main de l'homme. Mais les clichés de Salgado semblent être pris au cœur de l'enfer, les mineurs s'entassant comme dans un charnier et la préoccupation écologique que pourraient susciter ces photographies demeure secondaire par rapport à la représentation d'une catastrophe humanitaire.

12. Dans certaines expositions, d'autres clichés de Nolte sur le peuple Shawi ont été rajoutés à cette série initiale.

13. Texte du Musuk Nolte qui figurait sur son site personnel qui n'est plus opérationnel : http:// www.musuknolte.pe/, page consultée le 10 février 2017.

14. Nolte se distingue ainsi d'une pratique courante chez ses confrères. Rodrigo Rodrich, photojournaliste péruvien également très présent dans les expositions collectives amazoniennes, ne dissocie pas ses enquêtes menées pour El Comercio de son travail personnel. Son exemple est parlant puisqu'il a lui aussi réalisé un reportage sur le peuple shawi https:// rodrigorodrich.wordpress.com/tag/shawi/. On pourrait penser que cette séparation de la part de Nolte tiendrait d'une invalidation de la valeur documentaire des clichés de style plus artistique, mais il présente lui-même sa série Shawi comme documentaire.

15. Les récits de prise d'ayahuasca évoquent souvent un détachement du corps ou une transsubstantiation, notamment vers une figure animale. La littérature sur le sujet est abondante, voir par exemple Labate, Beatriz et Cavnar, Clancy, Ayahuasca Shamanism in the Amazon and Beyond, Oxford University Press, 2014.

16. L'ayahuasca est d'ailleurs pris dans les sociétés amazoniennes dans un but de sociabilité, entre communautés mais aussi envers les non-humains, qu'il s'agisse d'animaux ou de plantes.

17. Version non éditée de l'entretien de Musuk Nolte pour Bendayán, Christian (ed.), Amazonistas, op. cit.

\section{RÉSUMÉS}

Revendiquant une pratique entre le documentaire et l'artistique, déclarant travailler « depuis la pénombre » dans une esthétique de l'entre-deux, le photographe péruvien Musuk Nolte (Mexico, 1988) est l'un des rares de sa génération à connaître un succès équivalent dans ces deux domaines de la photographie. Ce trait est particulièrement visible dans ses séries amazoniennes, qu'elles documentent la coupe illégale d'arbres, un hôpital psychiatrique ou les effets de rituels chamaniques. Cet article propose de montrer comment, par cette posture, Nolte cherche à révéler des interstices encore invisibles d'un espace pourtant si photographié et suscitant une foule d'imaginaires topiques. Il se situe par ailleurs à contrecourant d'une tendance actuelle qui 
surenchérit une «tropicalité » criarde de l'Amazonie et d'une tendance historique du " goût de l'autre ».

Claiming that his activity unfolds between documentary and art, and stating that he works "from the shadows" within an aesthetic of interstice, Peruvian photographer Musuk Nolte (Mexico City, 1988) is one of the few of his generation to be equally successful in these two fields of photography. This aesthetic is particularly well featured in his photographs of Amazonia, whether they document illegal logging, the conditions of a psychiatric hospital or the effects of shamanistic rituals. This article seeks to establish how Nolte adopts this position in order to reveal what is still invisible in a space that has been abundantly photographed and that has spurred various topical imaginaries. One of his strategies is to go against a contemporary trend that focuses on a flashy "tropicality" of the rainforest and against a historical trend that cultivates the "taste for otherness".

Reivindicando una práctica entre lo documental y lo artístico y declarando trabajar "desde la penumbra" en una estética del intersticio, el fotógrafo peruano Musuk Nolte (Ciudad de México, 1988) es uno de los pocos de su generación en conocer un éxito equivalente en estos dos ámbitos de la fotografía. Este rasgo es particularmente patente en sus series amazónicas, sean estas documentales sobre la tala ilegal, sobre un hospital psiquiátrico o sobre los efectos de rituales chamánicos. Este artículo propone mostrar cómo, a través de esta postura, Nolte busca revelar los resquicios aún invisibles de un espacio muy fotografiado y que despierta una cantidad de imaginarios tópicos. Así, se sitúa a contracorriente de una tendencia actual de exagerar una "tropicalidad" estridente de la Amazonía y de una tendencia histórica del "gusto por lo otro".

\section{INDEX}

Palabras claves : Musuk Nolte, Amazonía, Perú, documental, fotografía

Keywords : Musuk Nolte, Amazonia, Peru, documentary, photography

Mots-clés : Musuk Nolte, Amazonie, Pérou, documentaire, photographie

\section{AUTEUR}

\section{MORGANA HERRERA}

Morgana Herrera est agrégée d'espagnol, ancienne élève de l'ENS de Lyon et diplômée de l'INALCO en quechua. Elle prépare actuellement une thèse à l'université de Toulouse Jean Jaurès sur l'inclusion de l'Amazonie dans la réflexion sur la culture nationale péruvienne dans les années 1940. Elle fait également partie de «Bufeo/Arte+Amazonía », galerie et espace de recherche sur l'art contemporain amazonien péruvien à Lima. morgana.herrera@univ-tlse2.fr 